\title{
Kinetics or Equilibrium? - A Commentary on a Recent Simulation Study of Semiochemical Dose-Response Curves of Insect Olfactory Sensing
}

\author{
Johannes W. R. Martini • Michael Habeck
}

Published online: 5 December 2014

(C) Springer Science+Business Media New York 2014

Olfactory and pheromone sensing is a fundamental process that controls insect behavior. It is therefore relevant to agriculture and forestry as well as epidemiology. However the mechanism of the signal transmission from the chemical signal to the electrophysiological response in insects is still not completely understood. There is an ongoing discussion about which class of parametric curves is most appropriate to describe the mechanism and to fit experimental measurements. Besides approaches based on ligand binding in equilibrium (e.g. Getz and Lánsky 2001) kinetic reaction curves have been used for data regression (e.g. Byers 2013). In this commentary, we analyze a stochastic model that has recently been used to simulate dose-response curves (Byers 2013). We show analytically, without the use of simulation and regression, that the resulting response curve will be a "first order" kinetic. Therefore, a regression analysis of the simulation data does not provide an independent hint that kinetic curves are the right parametric class to describe the electrophysiological response. Moreover, our analysis establishes a direct interpretation of the regression parameters in the model of Byers (2013).

This comment refers to the article available at doi:10.1007/s10886-0130328-6.

An author's reply to this comment is available at doi:10.1007/s10886014-0527-9.

J. W. R. Martini $(\bowtie) \cdot$ M. Habeck

Felix Bernstein Institute for Mathematical Statistics in the

Biosciences, University of Göttingen, Goldschmidtstrasse 7,

37077 Göttingen, Germany

e-mail: jmartin2@gwdg.de

J. W. R. Martini

Max Planck Institute for Developmental Biology, Spemannstr. 35-39,

72076 Tübingen, Germany

M. Habeck

Max Planck Institute for Biophysical Chemistry, Am Faßberg 11,

37077 Göttingen, Germany
The Model. Byers (2013) used the following model to simulate dose-response curves: The antenna is subdivided into $m$ segments of equal size among which are $N$ receptors. $n$ odorant molecules are shot at the antenna stochastically independently, with a uniform distribution. The dose response does not depend on how often a specific receptor is hit, but on how many receptors are hit at least once. The simulation was repeated eight times for 23 different ligand concentrations and regressed with a first order kinetic equation (as a function of the number of odorant molecules). We translate Byer's simulation into the following probabilistic model:

Let $X_{i}$ indicate whether the $i$-th receptor has been hit at least once $\left(X_{i}=1\right)$ or never $\left(X_{i}=0\right)$ by the $n$ odorant molecules. $X_{i}$ is a Bernoulli variable with $P_{1}\left(X_{i}=0\right)=\frac{m-1}{m}$ being the probability of missing the receptor in a single shot. Because all $n$ shots are independent, the probability of missing the receptor in $n$ shots is $P_{n}\left(X_{i}=0\right)=\left[P_{1}\left(X_{i}=0\right)\right]^{n}$.

Therefore, the $i$-th receptor will be hit with probability:

$P_{n}\left(X_{i}=1\right)=1-\mathrm{P}_{\mathrm{n}}\left(X_{i}=0\right)=1-\left(\frac{m-1}{m}\right)^{n}$

The simulation was repeated eight times for each odorant concentration and the regression curve should describe the average number of excited receptors as a function of the number of odorant molecules being "shot". Therefore, we are interested in the expectation of the sum of all Bernoulli variables $X=\sum_{i=1}^{N} X_{i}$, describing the number of receptors which are hit at least once. Because the expectation operator $E$ is linear (also if the variables $X_{i}$ are stochastically dependent), we obtain the following expression for the dose-response (note that the $X_{i}$ are identically distributed):

$\mathrm{EX}=\sum_{i=1}^{N} E X_{i}=\mathrm{NP}_{n}\left(X_{i}=1\right)=N\left[1-\left(\frac{m-1}{m}\right)^{n}\right]$ 
The right-hand site of Eq. (2) is a first order rate equation $a\left[1-e^{-b n}\right]$ and provides an interpretation of the regression parameters $a$ and $b$ :

$a=N$ and $b=\ln \left(\frac{m}{m-1}\right) \approx \frac{1}{m-1}$.

A comparison with Byer's regression analysis (see Fig. 3 in Byers (2013)) confirms our calculation. He obtained $a \approx 9999$ and $b \approx 1.002 \times 10^{-6}$ for a simulation with $N=10^{4}$ receptors and a total of $m=10^{6}$ segments.

The analytical view on the simulation also gives the variance $V$ of the response in this model:

$\mathrm{V}(\mathrm{X})=\sum_{i=1}^{N} V\left(X_{i}\right)+\sum_{i, j=1, i \neq j}^{N} \operatorname{Cov}\left(X_{i}, X_{j}\right)$

Because $X_{i}$ are Bernoulli variables, $V\left(X_{i}\right)=E X_{i}-\left(E X_{i}\right)^{2}$ and $\operatorname{Cov}\left(X_{i}, X_{j}\right)=E X_{i} X_{j}-E X_{i} E X_{j}$. Every term is known except for $E X_{i} X_{j}$. Moreover, since $X_{i}$ and $X_{j}$ are identically distributed Bernoulli variables, we have

$$
\begin{aligned}
E X_{i} X_{j} & =P_{n}\left(X_{i}=1, X_{j}=1\right) \\
& =1-2 P_{n}\left(X_{i}=1, X_{j}=0\right)-P_{n}\left(X_{i}=0, X_{j}=0\right) .
\end{aligned}
$$

The underlying stochastic model gives

$P_{n}\left(X_{i}=0, X_{j}=0\right)=\left(\frac{m-2}{m}\right)^{n}$

and

$$
\begin{aligned}
& P_{n}\left(X_{i}=1, X_{j}=0\right)=P_{n}\left(X_{i}=1 \mid X_{j}=0\right) \quad P_{n}\left(X_{j}=0\right) \\
& =\left(1-P_{n}\left(X_{i}=0, \mid X_{j}=0\right)\right) P_{n}\left(X_{j}=0\right)=\left[1-\left(\frac{m-2}{m-1}\right)^{n}\right]\left(\frac{m-1}{m}\right)^{n}
\end{aligned}
$$

and thus

$$
V(X)=N\left(\frac{m-1}{m}\right)^{n}-N^{2}\left(\frac{m-1}{m}\right)^{2 n}+N(N-1)\left(\frac{m-2}{m}\right)^{n} \cdot(4)
$$

For the sake of completeness, we would briefly like to mention that the stochastic model is an instance of the occupancy problem. Using results from Feller (1968) it is possible to derive the full distribution of the response:

$P_{n}(X=x)=\left(\begin{array}{l}N \\ x\end{array}\right) \sum_{i=0}^{x}(-1)^{i}\left(\begin{array}{l}x \\ i\end{array}\right)\left(1-\frac{N+i-x}{m}\right)^{n}$

where again $x=0,1, \ldots, N$ is the total number of receptors that have been hit at least once.

In this commentary, we calculated the dose-response curve resulting from a stochastic model proposed by Byers (2013) and confirmed that it is a first order kinetic. Moreover, we expressed the kinetic parameters through the parameters of the stochastic system. The functional form of the response curve is a direct consequence of the odorant molecules being "shot" independently at the antenna and the assumption that they move only in one direction. We therefore suggest an increased focus on the biology of odorant sensing in order to devise more detailed models of the process and to select the most appropriate class of response curves. One important aspect that should be incorporated in the model is the function of odorant-binding proteins (OBPs), which act as carriers to the receptor. Assuming that the odorant-OBP complexes move in all directions with the same probability, thermodynamic equilibrium equations based on the grand canonical partition function may be more adequate. A general problem with kinetic curves in this context is that although they might fit the experimental data well, they will not be able to explain how the response is produced. For example, could a kinetic curve predict how the dose-response curve changes if an OBP is down-regulated?

\section{References}

Byers JA (2013) Modeling and regression analysis of semiochemical dose-response curves of insect antennal reception and behavior. J Chem Ecol 39(8):1081-1089

Feller W (1968) An introduction to probability theory and its applications, 3rd edn. Wiley, New York

Getz WM, Lánsky P (2001) Receptor dissociationconstants and the information entropy of membranes coding ligand concentration. Chem Senses 26(2):95-104 